\title{
chis When One Diagnosis Reveals Another...
}

Heggie AJ, Nichols M, Simkiss L, Smith J, De-Alwis N, Carey P, Nayar R, Joshi A Sunderland Royal Hospital, Department of Diabetes, Endocrinology \& Metabolic Medicine, Sunderland

\section{Background}

Addison's disease is a relatively rare autoimmune condition that presents with non-specific symptoms or in crisis. Autoimmune hypothyroidism is a far more common disease, but can also present with non-specific symptoms. Adrenal insufficiency should be considered when symptoms do not improve in a patient with hypothyroidism who is commenced on levothyroxine.

\section{Case presentation}

- 37 year old man diagnosed with autoimmune hypothyroidism 2 months previously

- Episode of dizziness without syncope whilst operating a fork-lift truck at work

- Lost $10 \mathrm{~kg}$ in weight

\section{Findings}

- Postural drop in BP (lying 139/77mmHg, standing 115/76mmHg)

- TSH low with normal free T4

- Electrolytes typical of adrenal insufficiency, confirmed by SST (table 1)

- Raised ACTH and positive adrenal antibodies

\section{Management}

- Levothyroxine stopped

- Hydrocortisone and fludrocortisone started - Computed tomography demonstrated a small thymoma

-Acetylcholinesterase antibodies negative, cardiothoracic intervention not necessary

-TSH became mildly elevated with strongly positive TPO antibodies, so levothyroxine was re-commenced with good clinical effect.

\begin{tabular}{|c|c|c|c|}
\hline \multicolumn{3}{|l|}{ Biochemistry } & \\
\hline \multicolumn{3}{|l|}{$\mathrm{TSH}(\mathrm{mmol} / \mathrm{l})$} & 0.32 \\
\hline \multicolumn{3}{|l|}{ Free T4 $(p m o l / l)$} & 14.3 \\
\hline \multicolumn{3}{|l|}{ Sodium $(\mathrm{mmol} / \mathrm{l})$} & 130 \\
\hline \multicolumn{3}{|l|}{ Potassium $(\mathrm{mmol} / \mathrm{l})$} & 5.7 \\
\hline \multicolumn{3}{|l|}{ Bicarbonate $(\mathrm{mmol} / \mathrm{l})$} & 20 \\
\hline \multicolumn{4}{|l|}{ Short Synacthen test } \\
\hline & 0 mins & $30 \mathrm{mins}$ & $60 \mathrm{mins}$ \\
\hline $\operatorname{Cortisol}_{(n m o / /)}$ & 23 & 24 & 25 \\
\hline
\end{tabular}

Table 1. Biochemistry on presentation

\section{$\underline{\text { Discussion }}$}

- While studies have shown an increased risk of Addison's disease with Hashimoto's thyroiditis when compared to described background prevalence rates (1); interestingly a recent review of 3069 Caucasian patients with autoimmune hypothyroidism demonstrated only a near-significant association with Addison's disease when compared with age- and sex- matched controls (2).

-50\% of patients with Addison's will present in adrenal crisis (3), and other case reports describe significant weight loss in this situation (4). This case highlights the need to be aware of this relationship, and to consider adrenal insufficiency when symptoms do not improve on levothyroxine replacement, particularly with significant weight loss.

-This case is also an example of autoimmune polyglandular syndrome type 2 .

\section{Autoimmune polyglandular syndrome type 2}

Commonly manifests as adrenal insufficiency and autoimmune thyroid disease. May also involve type 1 diabetes mellitus and primary hypogonadism. Rarely manifests with vitiligo.

\section{References}

1. Boelaert K, et al. Prevalence and relative risk of other autoimmune diseases in subjects with autoimmune thyroid disease. Am J Med 2010; 123(2):183 e1-183.e9.

2. Fallahi $\mathrm{P}$, et al. The association of other autoimmune diseases in patients with autoimmune thyroiditis: Review of the literature and report of a large series of patients. Autoimmunity Reviews 2016; 15(12): 1125-8.

3. Chakera AJ, Vaidya B. Addison disease in adults: diagnosis and management.. Am J Med 2010; 123(5): 409413.

4. Choudry $\mathrm{N}$, et al. Thyroxine replacement precipitating adrenal crisis. Endocrine Abstracts 2009; 19:64. 